Article

\title{
Toric Rings and Ideals of Stable Set Polytopes
}

\author{
Kazunori Matsuda ${ }^{1}$, Hidefumi Ohsugi ${ }^{2, *}$ and Kazuki Shibata ${ }^{3}$ \\ 1 Faculty of Engineering, Kitami Institute of Technology, Kitami, Hokkaido 090-8507, Japan \\ 2 Department of Mathematical Sciences, School of Science and Technology, Kwansei Gakuin University, Sanda, \\ Hyogo 669-1337, Japan \\ 3 Department of Mathematics, College of Science, Rikkyo University, Toshima-ku, Tokyo 171-8501, Japan \\ * Correspondence: ohsugi@kwansei.ac.jp
}

Received: 6 June 2019; Accepted: 8 July 2019; Published: 10 July 2019

check for updates

\begin{abstract}
In the present paper, we study the normality of the toric rings of stable set polytopes, generators of toric ideals of stable set polytopes, and their Gröbner bases via the notion of edge polytopes of finite nonsimple graphs and the results on their toric ideals. In particular, we give a criterion for the normality of the toric ring of the stable set polytope and a graph-theoretical characterization of the set of generators of the toric ideal of the stable set polytope for a graph of stability number two. As an application, we provide an infinite family of stable set polytopes whose toric ideal is generated by quadratic binomials and has no quadratic Gröbner bases.
\end{abstract}

Keywords: toric ideals; Gröbner bases; graphs; stable set polytopes

\section{Introduction}

Let $\mathcal{P} \subset \mathbb{R}^{n}$ be an integral convex polytope, i.e., a convex polytope whose vertices have integer coordinates, and let $\mathcal{P} \cap \mathbb{Z}^{n}=\left\{\mathbf{a}_{1}, \ldots, \mathbf{a}_{m}\right\}$. Let $K\left[X, X^{-1}, t\right]=K\left[x_{1}, x_{1}^{-1}, \ldots, x_{n}, x_{n}^{-1}, t\right]$ be the Laurent polynomial ring in $n+1$ variables over a field $K$. Given an integer vector $\mathbf{a}=\left(a_{1}, \ldots, a_{n}\right) \in \mathbb{Z}^{n}$, we set $X^{\mathbf{a}} t=x_{1}^{a_{1}} \cdots x_{n}^{a_{n}} t \in K\left[X, X^{-1}, t\right]$. Then, the toric ring of $\mathcal{P}$ is the subalgebra $K[\mathcal{P}]$ of $K\left[X, X^{-1}, t\right]$ generated by $\left\{X^{\mathbf{a}_{1}} t, \ldots, X^{\mathbf{a}_{m}} t\right\}$ over $K$. Here, we need the variable $t$ in order to regard $K[\mathcal{P}]$ as a homogeneous algebra by setting each $\operatorname{deg} X^{\mathbf{a}_{i}} t=1$. The toric ideal $I_{\mathcal{P}}$ of $\mathcal{P}$ is the kernel of a surjective homomorphism $\pi: K\left[y_{1}, \ldots, y_{m}\right] \rightarrow K[\mathcal{P}]$ defined by $\pi\left(y_{i}\right)=X^{\mathbf{a}_{i}}$ for $1 \leq i \leq m$. In general, $I_{\mathcal{P}}$ is generated by homogeneous binomials and any reduced Gröbner basis of $I_{\mathcal{P}}$ consists of homogeneous binomials; see [1]. A simplex $\sigma$ is called a subsimplex of $\mathcal{P}$ if the set of vertices of $\sigma$ is contained in $\mathcal{P} \cap \mathbb{Z}^{n}$. A set $\Delta$ of subsimplices of $\mathcal{P}$ is called a covering of $\mathcal{P}$ if $\bigcup_{\sigma \in \Delta} \sigma=\mathcal{P}$. A covering $\Delta$ of $\mathcal{P}$ is called a triangulation of $\mathcal{P}$ if $\Delta$ is a simplicial complex. A covering (triangulation) $\Delta$ of $\mathcal{P}$ is called unimodular if the normalized volume of each maximal simplex in $\Delta$ is equal to 1 . The following properties of an integral convex polytope $\mathcal{P}$ have been investigated in many papers on commutative algebra and combinatorics:

(i) $\mathcal{P}$ is unimodular (any triangulation of $\mathcal{P}$ is unimodular), the initial ideal of $I_{\mathcal{P}}$ is generated by squarefree monomials with respect to any monomial order ([2] Section 4.3);

(ii) $\mathcal{P}$ is compressed (any "pulling" triangulation is unimodular), the initial ideal of $I_{\mathcal{P}}$ is generated by squarefree monomials with respect to any reverse lexicographic order [3,4];

(iii) $\mathcal{P}$ has a regular unimodular triangulation, there exists a monomial order such that the initial ideal of $I_{\mathcal{P}}$ is generated by squarefree monomials ([2] Theorem 4.17);

(iv) $\mathcal{P}$ has a unimodular triangulation ([2] Section 4.2.4);

(v) $\mathcal{P}$ has a unimodular covering ([2] Section 4.2.4);

(vi) $\mathcal{P}$ is normal, $K[\mathcal{P}]$ is a normal semigroup ring ([2] Section 4.2.3). 
Details for these conditions are explained in ([1] Chapter 8) and ([2] Chapter 4). The hierarchy (i) $\Rightarrow$ (ii) $\Rightarrow$ (iii) $\Rightarrow$ (iv) $\Rightarrow$ (v) is trivial by their definition. The implication (v) $\Rightarrow$ (vi) is explained in ([2] Theorem 4.11). Note that the converse of each of the above implications is false. On the other hand, the following properties of $I_{\mathcal{P}}$ are studied by many authors:

(a) $I_{\mathcal{P}}$ has a Gröbner bases consisting of quadratic binomials;

(b) $K[\mathcal{P}]$ is Koszul ([2] Definition 2.20);

(c) $I_{\mathcal{P}}$ is generated by quadratic binomials.

The hierarchy $(\mathrm{a}) \Rightarrow(\mathrm{b}) \Rightarrow$ (c) is known, and the converse of each of the two implications is false; see ([5] Examples 2.1 and 2.2) and [6].

The purpose of this paper is to study such properties of toric rings and ideals of stable set polytopes of simple graphs. Let $G$ be a finite simple graph on the vertex set $[n]=\{1,2, \ldots, n\}$, and let $E(G)$ denote the set of edges of $G$. Given a subset $W \subset[n]$, we associate the $(0,1)$ vector $\rho(W)=\sum_{j \in W} \mathbf{e}_{j} \in \mathbb{R}^{n}$, where $\mathbf{e}_{i}$ is the $i$ th unit vector of $\mathbb{R}^{n}$. In particular, $\rho(\varnothing)=\mathbf{0} \in \mathbb{R}^{n}$. A subset $W \subset[n]$ is said to be stable (or independent) if $\{i, j\} \notin E(G)$ for all $i, j \in W$ with $i \neq j$. In particular, $\varnothing$ and each $\{i\}$ with $i \in[n]$ are stable. Let $S(G)$ be the set of all stable sets of $G$. The stable set polytope (independent set polytope) $\mathcal{Q}_{G} \subset \mathbb{R}^{n}$ of a simple graph $G$ is the convex hull of $\{\rho(W): W \in S(G)\}$.

Example 1. If $G$ is a complete graph, then $\mathcal{Q}_{G}$ is a unit simplex, and hence $I_{\mathcal{Q}_{G}}=\{0\}$ and $K\left[\mathcal{Q}_{G}\right]=$ $K\left[x_{1} t, \ldots, x_{n} t, t\right] \simeq K\left[y_{1}, \ldots, y_{n+1}\right]$.

Stable set polytopes are very important in many areas, such as optimization theory as well as combinatorics and commutative algebra. Below, we present a list of results on the toric ring $K\left[\mathcal{Q}_{G}\right]$ and the toric ideals $I_{\mathcal{Q}_{G}}$ of the stable set polytope $\mathcal{Q}_{G}$ of a simple graph $G$.

1. The stable set polytope $\mathcal{Q}_{G}$ is compressed if and only if $G$ is perfect $([3,4,7])$.

2. Let $G$ be a perfect graph. Then, the toric ring $K\left[\mathcal{Q}_{G}\right]$ is Gorenstein if and only if all maximal cliques of $G$ have the same cardinality ([8]).

3. The toric ring $K\left[\mathcal{Q}_{G}\right]$ is strongly Koszul ([2] p. 53) if and only if $G$ is trivially perfect ([9] Theorem 5.1).

4. Let $G(P)$ be a comparability graph of a poset $P$. Then, $\mathcal{Q}_{G(P)}$ is called a chain polytope of $P$. It is known that the toric ideals of a chain polytope have a squarefree quadratic initial ideal (see [10] Corollary 3.1). For example, if a graph $G$ is bipartite, then there exists a poset $P$ such that $G=G(P)$.

5. Suppose that a graph $G$ on the vertex set $[n]$ is an almost bipartite graph, i.e., there exists a vertex $v$ such that the induced subgraph of $G$ on the vertex set $[n] \backslash\{v\}$ is bipartite. Then, $I_{\mathcal{Q}_{G}}$ has a squarefree quadratic initial ideal ([11] Theorem 8.1). For example, any cycle is almost bipartite.

6. Let $G$ be the complement of an even cycle of length $2 k$. Then, the maximum degree of a minimal set of binomial generators of $I_{\mathcal{Q}_{G}}$ is equal to $k$ ([11] Theorem 7.4).

In the present paper, we study the normality of the toric rings of stable set polytopes, generators of toric ideals of stable set polytopes, and their Gröbner bases via the notion of edge polytopes of finite (nonsimple) graphs and the results on their toric ideals. Here, the edge polytope $\mathcal{P}_{G} \subset \mathbb{R}^{n}$ of a graph $G$ allowing loops and having no multiple edges is the convex hull of

$$
\left\{\mathbf{e}_{i}+\mathbf{e}_{j}:\{i, j\} \text { is an edge of } G\right\} \cup\left\{2 \mathbf{e}_{i}: \text { there is a loop at } i \text { in } G\right\} .
$$

This paper is organized as follows. In Section 2, fundamental properties of $K\left[\mathcal{Q}_{G}\right]$ and $I_{\mathcal{Q}_{G}}$ are studied. In particular, the relationship between the stable set polytopes and the edge polytopes are given (Lemma 1). In addition, it is shown that $\mathcal{Q}_{G}$ is unimodular if and only if the complement of $G$ is bipartite (Proposition 5). We also point out that, by the results in [11], it is easy to see that $I_{\mathcal{Q}_{G}}$ 
has a squarefree quadratic initial ideal if $G$ is either a chordal graph or a ring graph (Proposition 2). In Section 3, we discuss the normality of the stable set polytopes. We prove that, for a simple graph $G$ of stability number two, $\mathcal{Q}_{G}$ is normal if and only if the complement of $G$ satisfies the "odd cycle condition" (Theorem 1). Using this criterion, we construct an infinite family of normal stable set polytopes without regular unimodular triangulations (Theorem 2). For general simple graphs, some necessary conditions for $\mathcal{Q}_{G}$ to be normal are also given. In Section 4 , we study the set of generators and Gröbner bases of toric ideals of stable set polytopes. It is shown that for a simple graph $G$ of stability number two, the set of binomial generators of $I_{\mathcal{Q}_{G}}$ are described in terms of even closed walks of a graph (Theorem 3). If $\bar{G}$ is bipartite and if $I_{\mathcal{Q}_{G}}$ is generated by quadratic binomials, then $I_{\mathcal{Q}_{G}}$ has a quadratic Gröbner basis (Corollary 1). Finally, using the results on normality, generators, and Gröbner bases, we present an infinite family of non-normal stable set polytopes whose toric ideal is generated by quadratic binomials and has no quadratic Gröbner bases (Theorem 4).

\section{Fundamental Properties of the Stable Set Polytopes}

In this section, we give some fundamental properties of $K\left[\mathcal{Q}_{G}\right]$ and $I_{\mathcal{Q}_{G}}$. In particular, a relation between the stable set polytopes and the edge polytopes is discussed. The stability number $\alpha(G)$ of a graph $G$ is the cardinality of the largest stable set. If a simple graph $G$ satisfies $\alpha(G)=1$, then $G$ is a complete graph in Example 1. Given lattice polytopes $\mathcal{P}_{1} \subset \mathbb{R}^{m}$ and $\mathcal{P}_{2} \subset \mathbb{R}^{n}$, the product of $\mathcal{P}_{1}$ and $\mathcal{P}_{2}$ is defined by $\mathcal{P}_{1} \times \mathcal{P}_{2}=\left\{\left(\alpha_{1}, \alpha_{2}\right) \in \mathbb{R}^{m+n}: \alpha_{1} \in \mathcal{P}_{1}, \alpha_{2} \in \mathcal{P}_{2}\right\}$. Then the toric ring $K\left[\mathcal{P}_{1} \times \mathcal{P}_{2}\right]$ is called the Segre product of $K\left[\mathcal{P}_{1}\right]$ and $K\left[\mathcal{P}_{2}\right]$.

Example 2. Suppose that a simple graph $G$ is not connected. Let $G_{1}, \ldots, G_{s}$ be the connected components of $G$. Then, it is easy to see that $\mathcal{Q}_{G}=\mathcal{Q}_{G_{1}} \times \cdots \times \mathcal{Q}_{G_{s}}$, and hence $K\left[\mathcal{Q}_{G}\right]$ is the Segre product of $K\left[\mathcal{Q}_{G_{1}}\right], \ldots, K\left[\mathcal{Q}_{G_{s}}\right]$.

Thus, it is enough to study stable set polytopes of connected simple graphs $G$ such that $\alpha(G) \geq 2$. We use the notion of toric fiber products to study toric rings of stable set polytopes. This notion is first introduced in [12] as a generalization of the Segre product. Since the definition of toric fiber products is complicated, we give an example.

Example 3. Let $G_{1}$ and $G_{2}$ be cycles of length 4 , where $E\left(G_{1}\right)=\{\{1,2\},\{2,3\},\{3,4\},\{1,4\}\}$ and $E\left(G_{2}\right)=\{\{3,4\},\{4,5\},\{5,6\},\{3,6\}\}$. Then $K\left[\mathcal{Q}_{G_{1}}\right]=K\left[t, x_{1} t, x_{2} t, x_{3} t, x_{4} t, x_{1} x_{3} t, x_{2} x_{4} t\right]$ and $K\left[\mathcal{Q}_{G_{2}}\right]=$ $K\left[t, x_{3} t, x_{4} t, x_{5} t, x_{6} t, x_{3} x_{5} t, x_{4} x_{6} t\right]$. We define the multigrading by $\operatorname{deg}\left(x_{1}^{a_{1}} \ldots x_{6}^{a_{6}} t^{\alpha}\right)=\left(a_{3}, a_{4}, \alpha\right)$. Let $A=$ $\{(0,0,1),(1,0,1),(0,1,1)\}$. Then, the toric fiber product $K\left[\mathcal{Q}_{G_{1}}\right] \times_{A} K\left[\mathcal{Q}_{G_{2}}\right]$ is generated by the monomials $x_{1}^{a_{1}} \ldots x_{4}^{a_{4}} t^{\alpha} \cdot x_{3}^{b_{3}} \ldots x_{6}^{b_{6}} t^{\beta}$ such that $x_{1}^{a_{1}} \ldots x_{4}^{a_{4}} t^{\alpha} \in K\left[\mathcal{Q}_{G_{1}}\right]$ and $x_{3}^{b_{3}} \ldots x_{6}^{b_{6}} t^{\beta} \in K\left[\mathcal{Q}_{G_{2}}\right]$ have the same multidegree a $\in A$. It is easy to see that $K\left[\mathcal{Q}_{G_{1}}\right] \times_{A} K\left[\mathcal{Q}_{G_{2}}\right] \cong K\left[\mathcal{Q}_{G_{1} \cup G_{2}}\right]$, and A corresponds to the lattice point in $\mathcal{Q}_{G_{1} \cap G_{2}}$, which is a simplex.

The application of toric fiber products to toric rings of stable set polytopes was studied in [11]. For $i=1,2$, let $G_{i}$ be a simple graph on the vertex set $V_{i}$ and the edge set $E_{i}$. If $V_{1} \cap V_{2}$ is a clique of both $G_{1}$ and $G_{2}$, then we construct a new graph $G_{1} \sharp G_{2}$ on the vertex set $V_{1} \cup V_{2}$ and the edge set $E_{1} \cup E_{2}$, which is called the clique sum of $G_{1}$ and $G_{2}$ along $V_{1} \cap V_{2}$.

Proposition 1. Let $G_{1} \sharp G_{2}$ be the clique sum of simple graphs $G_{1}$ and $G_{2}$. Then, $I_{\mathcal{Q}_{G_{1} \sharp G_{2}}}$ is a toric fiber product of $I_{\mathcal{Q}_{G_{1}}}$ and $I_{\mathcal{Q}_{G_{2}}}$. We can construct a set of binomial generators (or a Gröbner basis) of $I_{\mathcal{Q}_{G_{1} \sharp G_{2}}}$ from that of $I_{\mathcal{Q}_{G_{i}}}$ 's and some quadratic binomials. Moreover, $K\left[\mathcal{Q}_{G_{1} \sharp G_{2}}\right]$ is normal if and only if both $K\left[\mathcal{Q}_{G_{1}}\right]$ and $K\left[\mathcal{Q}_{G_{2}}\right]$ are normal.

Proof. Note that $I_{\mathcal{Q}_{G_{1} \cap G_{2}}}=\{0\}$. Hence, this is a special case of ([11] Proposition 5.1) by ([11] Proposition 9.6). 
A simple graph $G$ is called chordal if any induced cycle of $G$ is of length 3. A graph $G$ is called a ring graph if each block of $G$ that is not a bridge or a vertex can be constructed from a cycle by successively adding cycles of length $\geq 3$ using the clique sum construction. Ring graphs are introduced in $[13,14]$.

Proposition 2. Suppose that a simple graph $G$ is either a chordal graph or a ring graph. Then, $I_{\mathcal{Q}_{G}}$ has a squarefree quadratic initial ideal.

Proof. It is known by ([15] Proposition 5.5.1) that a graph $G$ is chordal if and only if $G$ is a clique sum of complete graphs. By the statement in Example 1 and Proposition $1, I_{\mathcal{Q}_{G}}$ has a squarefree quadratic initial ideal if $G$ is chordal.

Suppose that $G$ is a ring graph. Then, $G$ is a clique sum of trees and cycles since any graph is a clique sum (along a vertex) of trees and its blocks. Since trees and cycles are almost bipartite, by ([11] Theorem 8.1), the toric ideal $I_{\mathcal{Q}_{H}}$ has a squarefree quadratic initial ideal if $H$ is either a tree or a cycle. Thus, by Proposition $1, I_{\mathcal{Q}_{G}}$ has a squarefree quadratic initial ideal if $G$ is a ring graph.

A graph $G$ is called perfect if the chromatic number of every induced subgraph of $G$ is equal to the size of the largest clique of that subgraph; see [15]. We recall the following result on perfect graphs and their stable set polytopes; see $[3,4,7]$.

Proposition 3. Let $G$ be a simple graph. Then, $\mathcal{Q}_{G}$ is compressed if and only if $G$ is perfect. In particular, if $G$ is perfect, then $\mathcal{Q}_{G}$ is normal.

For a graph $G$ on the vertex set $[n]$, let $\bar{G}$ denote the complement of a graph $G$. An induced cycle of $G$ of length $>3$ is called a hole of $G$ and an induced cycle of $\bar{G}$ of length $>3$ is called an antihole of $G$. Below we combine two important characterizations of perfect graphs, where the first part is the strong perfect graph theorem and the second part considers just the perfect graphs with stability number 2 .

Proposition 4. Let $G$ be a simple graph. Then $G$ is a perfect graph if and only if $G$ has no odd holes and no odd antiholes. In particular, $G$ is a perfect graph with $\alpha(G)=2$ if and only if $\bar{G}$ is bipartite and not empty.

For a graph $G$, let $\bar{G}^{\star}$ be the nonsimple graph on the vertex set $[n+1]$ whose edge (and loop) set is $E(\bar{G}) \cup\{\{i, n+1\}: i \in[n+1]\}$. The following lemma plays an important role when we study the stable set polytope $\mathcal{Q}_{G}$ of $G$.

Lemma 1. Let $G$ be a simple graph with $\alpha(G)=2$. Then we have $K\left[\mathcal{Q}_{G}\right] \simeq K\left[\mathcal{P}_{\bar{G}^{\star}}\right]$. Moreover, if $\bar{G}$ is bipartite, then there exists a bipartite graph $H$ such that $K\left[\mathcal{Q}_{G}\right] \simeq K\left[\mathcal{P}_{H}\right]$.

Proof. Let $\varphi: K\left[\mathcal{Q}_{G}\right] \rightarrow K\left[x_{1}, \ldots, x_{n+1}\right]$ be the injective ring homomorphism defined by

$$
\varphi\left(x_{1}^{a_{1}} \cdots x_{n}^{a_{n}} t\right)=x_{1}^{a_{1}} \cdots x_{n}^{a_{n}} x_{n+1}^{2-\left(a_{1}+\cdots+a_{n}\right)} .
$$

Then $\varphi(t)=x_{n+1}^{2}, \varphi\left(x_{i} t\right)=x_{i} x_{n+1}$ for $i=1,2, \ldots, n$, and $\varphi\left(x_{k} x_{\ell} t\right)=x_{k} x_{\ell}$ for each stable set $\{k, \ell\}$ of $G$. Note that $\{k, \ell\}$ is a stable set of $G$ if and only if $\{k, \ell\}$ is an edge of $\bar{G}$. Hence, the image of $\varphi$ is $K\left[\mathcal{P}_{\bar{G}^{\star}}\right]$.

Suppose that $\bar{G}$ is bipartite. Then $\bar{G}$ has no odd cycles. Hence, any odd cycle of $\bar{G}^{\star}$ have the vertex $n+1$. (Note that $\{n+1, n+1\}$ is an odd cycle of length 1.) Thus, in particular, any two odd cycles of $\bar{G}^{\star}$ has a common vertex. We now show that there exists a bipartite graph $H$ such that $K\left[\mathcal{P}_{\bar{G}^{\star}}\right] \simeq K\left[\mathcal{P}_{H}\right]$ (by a similar argument in ([16] Proof of Proposition 5.5)). Let $[n]=V_{1} \cup V_{2}$ be a partition of the vertex set of the bipartite graph $\bar{G}$. Let $H$ be a bipartite graph on the vertex set $[n+2]$ and the edge set

$$
E(H)=E(\bar{G}) \cup\left\{\{i, n+1\}: i \in V_{1}\right\} \cup\left\{\{j, n+2\}: j \in V_{2}\right\} \cup\{\{n+1, n+2\}\} .
$$


Let $\psi: K\left[\mathcal{P}_{H}\right] \rightarrow K\left[x_{1}, \ldots, x_{n+1}\right]$ be the ring homomorphism defined by

$$
\psi\left(x_{1}^{a_{1}} \cdots x_{n+2}^{a_{n+2}}\right)=x_{1}^{a_{1}} \cdots x_{n}^{a_{n}} x_{n+1}^{a_{n+1}+a_{n+2}} .
$$

Since $\bar{G}^{\star}$ is obtained from $H$ by identifying the vertices $n+1$ and $n+2$, it follows that the image of $\psi$ is $K\left[\mathcal{P}_{\bar{G}^{\star}}\right]$. Hence, it is enough to show that $\psi$ is injective. Suppose that $u=x_{1}^{a_{1}} \cdots x_{n+2}^{a_{n+2}}$, $v=x_{1}^{b_{1}} \cdots x_{n+2}^{b_{n+2}} \in K\left[\mathcal{P}_{H}\right]$ satisfies $\psi(u)=\psi(v)$. Then $a_{n+1}+a_{n+2}=b_{n+1}+b_{n+2}$ and $a_{i}=b_{i}$ for $i=1,2, \ldots, n$. Since $[n]=V_{1} \cup V_{2}$ is a partition for $\bar{G}$, we have

$$
a_{n+2}+\sum_{k \in V_{1}} a_{k}=a_{n+1}+\sum_{\ell \in V_{2}} a_{\ell}, \quad b_{n+2}+\sum_{k \in V_{1}} b_{k}=b_{n+1}+\sum_{\ell \in V_{2}} b_{\ell}
$$

Thus, $a_{n+1}=b_{n+1}$ and $a_{n+2}=b_{n+2}$, as desired.

The first application of Lemma 1 is as follows:

Proposition 5. Let $G$ be a simple graph. Then the following conditions are equivalent:

(i) $\mathcal{Q}_{G}$ is unimodular;

(ii) $\bar{G}$ is bipartite.

Moreover, if $\alpha(G)=2$, then the conditions

(iii) $\mathcal{Q}_{G}$ is compressed;

(iv) $G$ is perfect

are also equivalent to conditions (i) and (ii).

Proof. We may assume that $G$ is not complete (i.e., $\bar{G}$ is not empty and $\alpha(G) \neq 1$ ). Let $A$ be the matrix whose columns are vertices of $\mathcal{Q}_{G}$, and let

$$
\widetilde{A}=\left(\begin{array}{rrr} 
& A & \\
1 & \cdots & 1
\end{array}\right) \text { and } B=\left(\begin{array}{cccc}
\mathbf{0} & \mathbf{e}_{1} & \cdots & \mathbf{e}_{n} \\
1 & 1 & \cdots & 1
\end{array}\right)
$$

Then $B$ is a submatrix of $\widetilde{A}$. Since $|\operatorname{det}(B)|=1$, the rank of $\widetilde{A}$ is $n+1$. It is known by ([1] p. 70) that $\mathcal{Q}_{G}$ is unimodular if and only if the absolute value of any nonzero $(n+1)$-minor of the matrix $\widetilde{A}$ is 1 .

Suppose that $\bar{G}$ is not bipartite. Then $\bar{G}$ has an odd cycle $C=\left(i_{1}, \ldots, i_{2 \ell+1}\right)$. Then the absolute value of the $(n+1)$-minor of $\widetilde{A}$ that corresponds to

$$
\left\{\mathbf{e}_{i_{k}}+\mathbf{e}_{i_{k+1}}: 1 \leq k \leq 2 \ell\right\} \cup\left\{\mathbf{e}_{i_{1}}+\mathbf{e}_{i_{2 \ell+1}}, \mathbf{0}\right\} \cup\left\{\mathbf{e}_{j}: j \notin\left\{i_{1}, \ldots, i_{2 \ell+1}\right\}\right\}
$$

equals 2. Hence, $\mathcal{Q}_{G}$ is not unimodular. Thus, we have (i) $\Rightarrow$ (ii).

Suppose that $\bar{G}$ is bipartite. By Lemma 1, there exists a bipartite graph $H$ such that $K\left[\mathcal{Q}_{G}\right] \simeq K\left[\mathcal{P}_{H}\right]$. It is well known that the edge polytope of a bipartite graph is unimodular; see ([2] Theorem 5.24). Thus, $\mathcal{P}_{H}$ is unimodular, and hence we have (ii) $\Rightarrow$ (i).

Suppose that $\alpha(G)=2$. By Proposition 3, conditions (iii) and (iv) are equivalent. In addition, by Proposition 4, conditions (ii) and (iv) are equivalent.

We close this section with the following fundamental fact on stable set and edge polytopes.

Proposition 6. Let $G^{\prime}$ be an induced subgraph of a graph $G$. Then

(i) the edge polytope $\mathcal{P}_{G^{\prime}}$ is a face of $\mathcal{P}_{G}$;

(ii) if $G$ is a simple graph, then $\mathcal{Q}_{G^{\prime}}$ is a face of $\mathcal{Q}_{G}$. 
It can be seen from Proposition 6 that several properties of $K\left[\mathcal{Q}_{G}\right]$ (resp. $K\left[\mathcal{P}_{G}\right]$ ) are inherited to $K\left[\mathcal{Q}_{G^{\prime}}\right]$ (resp. $K\left[\mathcal{P}_{G^{\prime}}\right]$ ), for example, normality of the toric ring, the existence of a squarefree initial ideal, existence of a quadratic Gröbner basis, and the existence of the set of quadratic binomial generators of the toric ideal; see [17].

\section{Normality of Stable Set Polytopes}

In this section, we study the normality of stable set polytopes. Normality of edge polytopes is studied in [18,19], and we make use of the normality conditions of edge polytopes while working with stable set polytopes, due to the relation discovered in Lemma 1. If $C_{1}$ and $C_{2}$ are cycles in a graph $G$, then $\{i, j\} \in E(G)$ is called a bridge of $C_{1}$ and $C_{2}$ if $i$ is a vertex of $C_{1} \backslash C_{2}$ and $j$ is a vertex of $C_{2} \backslash C_{1}$. We say that a graph $G$ satisfies the odd cycle condition if any induced odd cycles $C_{1}$ and $C_{2}$ in $G$ have either a common vertex or a bridge. For the sake of simplicity, assume that a graph $H$ has at most one loop. Then, it is known by $[18,19]$ that $\mathcal{P}_{H}$ is normal if and only if each connected component of $H$ satisfies the odd cycle condition. By ([18] Corollary 2.3) and Lemma 1, we have the following. (Note that $\bar{G}$ below is not necessarily connected.)

Theorem 1. Let $G$ be a simple graph with $\alpha(G)=2$. Then the following conditions are equivalent.

(i) $\mathcal{Q}_{G}$ is normal;

(ii) $\mathcal{Q}_{G}$ has a unimodular covering;

(iii) $\bar{G}$ satisfies the odd cycle condition, i.e., if two odd holes $C_{1}$ and $C_{2}$ in $\bar{G}$ have no common vertices, then there exists a bridge of $C_{1}$ and $C_{2}$ in $\bar{G}$.

In particular, if $\mathcal{Q}_{G}$ is normal, then $\mathcal{P}_{\bar{G}}$ is normal.

Proof. By Lemma 1, we have $K\left[\mathcal{Q}_{G}\right] \simeq K\left[\mathcal{P}_{\bar{G}^{\star}}\right]$. Hence, by ([18] Corollary 2.3), conditions (i) and (ii) are equivalent, and they hold if and only if $\bar{G}^{\star}$ satisfies the odd cycle condition. (Note that $\bar{G}^{\star}$ is connected.) Since the vertex $n+1$ is incident to any vertex of $\bar{G}^{\star}$, it is easy to see that $\bar{G}^{\star}$ satisfies the odd cycle condition if and only if $\bar{G}$ satisfies the odd cycle condition.

It is shown in [20] that there exists a graph $G$ such that $\mathcal{P}_{G}$ is normal and that $I_{\mathcal{P}_{G}}$ has no squarefree initial ideals. Examples on infinite families of such edge polytopes are given in [21]. We can construct the stable set polytopes with the same properties. Let $G_{1}\left(p_{1}, \ldots, p_{5}\right)$ be the graph defined in ([21] Theorem 3.10).

Theorem 2. Let $G$ be a graph such that $\bar{G}=G_{1}\left(p_{1}, \ldots, p_{5}\right)$ with $p_{i} \geq 2$ for $i=1, \ldots, 5$. Then $\mathcal{Q}_{G}$ is normal, and $I_{\mathcal{Q}_{G}}$ has no squarefree initial ideals.

Proof. Since $\bar{G}$ has no triangles, we have $\alpha(G)=2$. Since $G_{1}\left(p_{1}, \ldots, p_{5}\right)$ satisfies the odd cycle condition, $\mathcal{Q}_{G}$ is normal by Theorem 1 . On the other hand, $I_{\bar{G}}$ has no squarefree initial ideals. Since $\bar{G}$ is an induced subgraph of $\bar{G}^{\star}, I_{\mathcal{Q}_{G}}$ has no squarefree initial ideals by Lemma 1 and Proposition 6.

It seems to be a challenging problem to characterize the normal stable set polytopes with large stability numbers. We give several necessary conditions. The following is a consequence of Proposition 6 and Theorem 1.

Proposition 7. Let $G$ be a simple graph. Suppose that $\mathcal{Q}_{G}$ is normal. Then any two odd holes of $\bar{G}$ without a common vertex have a bridge in $\bar{G}$.

Proof. Suppose that two odd holes $C_{1}, C_{2}$ of $\bar{G}$ without common vertices have no bridges in $\bar{G}$. Let $H$ be an induced subgraph of $G$ whose vertex set is that of $C_{1} \cup C_{2}$. Then $\alpha(H)=2$, and hence $\mathcal{Q}_{H}$ is not normal by Theorem 1 . Thus, $\mathcal{Q}_{G}$ is not normal by Proposition 6 . 
Similar conditions are required for antiholes of $\bar{G}$.

Proposition 8. Let $G$ be a simple graph. Suppose that $\mathcal{Q}_{G}$ is normal. Then $G$ satisfies all of the following conditions:

(i) Any two odd antiholes of $\bar{G}$ having no common vertices have a bridge in $\bar{G}$.

(ii) Any two odd antiholes of $\bar{G}$ of length $\geq 7$ having exactly one common vertex have a bridge in $\bar{G}$.

(iii) Any odd hole and odd antihole of $\bar{G}$ having no common vertices have a bridge in $\bar{G}$.

Proof. Let $G$ be a graph on the vertex set $[n]$. Let $\mathcal{A}=\{(\rho(W), 1): W \in S(G)\}$. It is known by ([1] Proposition 13.5) that $\mathcal{Q}_{G}$ is normal if and only if we have $\mathbb{Z}_{\geq 0} \mathcal{A}=\mathbb{Q}_{\geq 0} \mathcal{A} \cap \mathbb{Z}^{n+1}$.

(i) Let $C_{1}=\left(i_{1}, \ldots, i_{2 k+1}\right)$ and $C_{2}=\left(j_{1}, \ldots, j_{2 \ell+1}\right)$ be odd antiholes in $\bar{G}$ having no common vertices and no bridges in $\bar{G}$. By Proposition 6, we may assume that $\bar{G}=C_{1} \cup C_{2}$. Then,

$$
\begin{aligned}
\sum_{W \in S\left(\overline{C_{1}}\right) \text { and }|W|=k}(\rho(W), 1) & =(2 k+1) \mathbf{e}_{n+1}+k \sum_{p=1}^{2 k+1} \mathbf{e}_{i_{p}}, \\
\sum_{W \in S\left(\overline{C_{2}}\right) \text { and }|W|=\ell}(\rho(W), 1) & =(2 \ell+1) \mathbf{e}_{n+1}+\ell \sum_{q=1}^{2 \ell+1} \mathbf{e}_{j_{q}} .
\end{aligned}
$$

Since $k, \ell \geq 2$, we have $k \ell-k-\ell \geq 0$. Hence,

$$
\begin{aligned}
\alpha & :=5 \mathbf{e}_{n+1}+\sum_{p=1}^{2 k+1} \mathbf{e}_{i_{p}}+\sum_{q=1}^{2 \ell+1} \mathbf{e}_{j_{q}} \\
& =\frac{1}{k}\left((2 k+1) \mathbf{e}_{n+1}+k \sum_{p=1}^{2 k+1} \mathbf{e}_{i_{p}}\right)+\frac{1}{\ell}\left((2 \ell+1) \mathbf{e}_{n+1}+\ell \sum_{q=1}^{2 \ell+1} \mathbf{e}_{j_{q}}\right)+\frac{k \ell-k-\ell}{k \ell} \mathbf{e}_{n+1}
\end{aligned}
$$

belongs to $\mathbb{Q}_{\geq 0} \mathcal{A} \cap \mathbb{Z}^{n+1}$. Suppose that $\alpha$ belongs to $\mathbb{Z}_{\geq 0} \mathcal{A}$. Since the $(n+1)$-th coordinate of $\alpha$ is 5 , there exist $W_{1}, \ldots, W_{5}$ such that

$$
\alpha=\left(\rho\left(W_{1}\right), 1\right)+\cdots+\left(\rho\left(W_{5}\right), 1\right),
$$

where each $W_{i}$ belongs to either $S\left(\overline{C_{1}}\right)$ or $S\left(\overline{C_{2}}\right)$. It then follows that $\sum_{W_{i} \in S\left(\overline{C_{1}}\right)}\left|W_{i}\right|=2 k+1$ and $\sum_{W_{i} \in S\left(\overline{C_{2}}\right)}\left|W_{i}\right|=2 \ell+1$. Since $\max \left\{|W|: W \in S\left(\overline{C_{1}}\right)\right\}=k, \max \left\{|W|: W \in S\left(\overline{C_{2}}\right)\right\}=\ell$, we have

$$
\begin{aligned}
& \left|\left\{W_{1}, \ldots, W_{5}\right\} \cap S\left(\overline{C_{1}}\right)\right| \geq 3 \\
& \left|\left\{W_{1}, \ldots, W_{5}\right\} \cap S\left(\overline{C_{2}}\right)\right| \geq 3 .
\end{aligned}
$$

This is a contradiction. Thus, $\alpha$ is not in $\mathbb{Z}_{\geq 0} \mathcal{A}$.

(ii) Let $C_{1}=\left(i_{1}, \ldots, i_{2 k+1}\right)$ and $C_{2}=\left(j_{1}, \ldots, j_{2 \ell+1}\right)$ be odd antiholes in $\bar{G}$ of length $\geq 7$ having exactly one common vertex $i_{1}=j_{1}$ and no bridges in $\bar{G}$. By Proposition 6 , we may assume that $\bar{G}=C_{1} \cup C_{2}$. Let

$$
\begin{aligned}
& S_{1}=\left\{W \in S\left(\overline{C_{1}}\right):|W|=k \text { and either } i_{1} \in W \text { or }\left\{i_{2}, i_{2 k+1}\right\} \subset W\right\} \\
& S_{2}=\left\{W \in S\left(\overline{C_{2}}\right):|W|=\ell \text { and either } j_{1} \in W \text { or }\left\{j_{2}, j_{2 \ell+1}\right\} \subset W\right\} .
\end{aligned}
$$


Then,

$$
\begin{aligned}
\sum_{W \in S_{1}}(\rho(W), 1) & =(2 k-1) \mathbf{e}_{n+1}+k \mathbf{e}_{i_{1}}+(k-1) \sum_{p=2}^{2 k+1} \mathbf{e}_{i_{p}}, \\
\sum_{W \in S_{2}}(\rho(W), 1) & =(2 \ell-1) \mathbf{e}_{n+1}+\ell \mathbf{e}_{i_{1}}+(\ell-1) \sum_{q=2}^{2 \ell+1} \mathbf{e}_{j_{q}} .
\end{aligned}
$$

Since $k, \ell \geq 3$, we have $0<1 /(k-1)+1 /(\ell-1) \leq 1$. Hence,

$$
\begin{aligned}
\alpha:= & 5 \mathbf{e}_{n+1}+3 \mathbf{e}_{i_{1}}+\sum_{p=2}^{2 k+1} \mathbf{e}_{i_{p}}+\sum_{q=2}^{2 \ell+1} \mathbf{e}_{j_{q}} \\
= & \frac{1}{k-1}\left((2 k-1) \mathbf{e}_{n+1}+k \mathbf{e}_{i_{1}}+(k-1) \sum_{p=2}^{2 k+1} \mathbf{e}_{i_{p}}\right) \\
& +\frac{1}{\ell-1}\left((2 \ell-1) \mathbf{e}_{n+1}+\ell \mathbf{e}_{i_{1}}+(\ell-1) \sum_{q=2}^{2 \ell+1} \mathbf{e}_{j_{q}}\right)+\left(1-\frac{1}{k-1}-\frac{1}{\ell-1}\right)\left(\mathbf{e}_{i_{1}}+\mathbf{e}_{n+1}\right)
\end{aligned}
$$

belongs to $\mathbb{Q}_{\geq 0} \mathcal{A} \cap \mathbb{Z}^{n+1}$. Since the $(n+1)$-th coordinate of $\alpha$ is 5 , there exist $W_{1}, \ldots, W_{5} \in S(\bar{G})$ such that $\alpha=\left(\rho\left(W_{1}\right), 1\right)+\cdots+\left(\rho\left(W_{5}\right), 1\right)$. Then, each $W_{i}$ belongs to either $S\left(\overline{C_{1}}\right)$ or $S\left(\overline{C_{2}}\right)$. Since $\max \{|W|$ : $\left.W \in S\left(\overline{C_{1}}\right)\right\}=k, \max \left\{|W|: W \in S\left(\overline{C_{2}}\right)\right\}=\ell$, we have

$$
\begin{aligned}
& \left|\left\{W_{1}, \ldots, W_{5}\right\} \cap S\left(\overline{C_{1}}\right)\right| \geq 2, \\
& \left|\left\{W_{1}, \ldots, W_{5}\right\} \cap S\left(\overline{C_{2}}\right)\right| \geq 2 .
\end{aligned}
$$

Thus, $\left(\left|\left\{W_{1}, \ldots, W_{5}\right\} \cap S\left(\overline{C_{1}}\right)\right|,\left|\left\{W_{1}, \ldots, W_{5}\right\} \cap S\left(\overline{C_{2}}\right)\right|\right)$ is either $(2,3)$ or $(3,2)$. Changing indices if necessary, we may assume that $W_{1}, W_{2} \in S\left(\overline{C_{1}}\right)$ and $W_{3}, W_{4}, W_{5} \in S\left(\overline{C_{2}}\right)$. It then follows that $\rho\left(W_{1}\right)+\rho\left(W_{2}\right)=\sum_{p=2}^{2 k+1} \mathbf{e}_{i_{p}}$, and hence $\rho\left(W_{3}\right)+\rho\left(W_{4}\right)+\rho\left(W_{5}\right)=3 \mathbf{e}_{i_{1}}+\sum_{q=2}^{2 \ell+1} \mathbf{e}_{j_{q}}$. This implies that $i_{1} \in W_{3} \cap W_{4} \cap W_{5}$. Thus, $i_{2}, i_{2 \ell+1} \notin W_{3}, W_{4}, W_{5}$, a contradiction. Therefore, we have $\alpha \notin \mathbb{Z}_{\geq 0} \mathcal{A}$.

(iii) Let $C_{1}=\left(i_{1}, \ldots, i_{2 k+1}\right)$ be an odd hole and $C_{2}=\left(j_{1}, \ldots, j_{2 \ell+1}\right)$ an odd antihole in $\bar{G}$ having no common vertices. By Proposition 6, we may assume that $\bar{G}=C_{1} \cup C_{2}$. Then,

$$
\begin{aligned}
\sum_{W \in S\left(\overline{C_{1}}\right) \text { and }|W|=2}(\rho(W), 1) & =(2 k+1) \mathbf{e}_{n+1}+2 \sum_{p=1}^{2 k+1} \mathbf{e}_{i_{p}} \\
\sum_{W \in S\left(\overline{C_{2}}\right) \text { and }|W|=\ell}(\rho(W), 1) & =(2 \ell+1) \mathbf{e}_{n+1}+\ell \sum_{q=1}^{2 \ell+1} \mathbf{e}_{j_{q}} .
\end{aligned}
$$

Hence,

$$
\begin{aligned}
& (k+3) \mathbf{e}_{n+1}+\sum_{p=1}^{2 k+1} \mathbf{e}_{i_{p}}+\sum_{q=1}^{2 \ell+1} \mathbf{e}_{j_{q}} \\
= & \frac{1}{2}\left((2 k+1) \mathbf{e}_{n+1}+2 \sum_{p=1}^{2 k+1} \mathbf{e}_{i_{p}}\right)+\frac{1}{\ell}\left((2 \ell+1) \mathbf{e}_{n+1}+\ell \sum_{q=1}^{2 \ell+1} \mathbf{e}_{j_{q}}\right)+\frac{\ell-2}{2 \ell} \mathbf{e}_{n+1}
\end{aligned}
$$

belongs to $\mathbb{Q}_{\geq 0} \mathcal{A} \cap \mathbb{Z}^{n+1}$. However, this vector is not in $\mathbb{Z}_{\geq 0} \mathcal{A}$ since $\max \left\{|W|: W \in S\left(\overline{C_{1}}\right)\right\}=2$, $\max \left\{|W|: W \in S\left(\overline{C_{2}}\right)\right\}=\ell$, and $\lceil(2 k+1) / 2\rceil+\lceil(2 \ell+1) / \ell\rceil=k+4>k+3$.

Unfortunately, the above conditions are not sufficient to be normal in general. For example, if the length of the two odd antiholes of $\bar{G}$ without common vertices are long, then a lot of bridges in $\bar{G}$ seem to be needed. 


\section{Generators and Gröbner Bases of $I_{\mathcal{Q}_{G}}$}

For a toric ideal $I$, let $d(I)$ be the maximum degree of binomials in a minimal set of binomial generators of $I$. If $I=\{0\}$, then we set $d(I)=0$. In this section, we study $d\left(I_{\mathcal{Q}_{G}}\right)$ by using results on the toric ideals of edge polytopes.

Let $G$ be a graph on the vertex set $[n]$ allowing loops and having no multiple edges. Let $E(G)=$ $\left\{e_{1}, \ldots, e_{m}\right\}$ be a set of all edges and loops of $G$. The toric ideal $I_{\mathcal{P}_{G}}$ is the kernel of a homomorphism $\pi: K\left[y_{1}, \ldots, y_{m}\right] \rightarrow K\left[x_{1}, \ldots, x_{n}\right]$ defined by $\pi\left(y_{i}\right)=x_{k} x_{\ell}$ where $e_{i}=\{k, \ell\}$. A finite sequence of the form

$$
\Gamma=\left(\left\{v_{1}, v_{2}\right\},\left\{v_{2}, v_{3}\right\}, \ldots,\left\{v_{q}, v_{q+1}\right\}\right)
$$

with each $\left\{v_{k}, v_{k+1}\right\} \in E(G)$ is called a walk of length $q$ of $G$ connecting $v_{1} \in[n]$ and $v_{q+1} \in[n]$. A walk $\Gamma$ of the form (1) is called even (resp. odd) if $q$ is even (resp. odd). A walk $\Gamma$ of the form (1) is called closed if $v_{q+1}=v_{1}$. Given an even closed walk $\Gamma=\left(e_{i_{1}}, e_{i_{2}}, \ldots, e_{i_{2 q}}\right)$ of $G$, we write $f_{\Gamma}$ for the binomial

$$
f_{\Gamma}=\prod_{k=1}^{q} y_{i_{2 k-1}}-\prod_{k=1}^{q} y_{i_{2 k}} \in I_{\mathcal{P}_{G}} .
$$

We regard a loop as an odd cycle of length 1 . We recall the following result from $[1,5,22]$.

Proposition 9. Let $G$ be a graph having at most one loop. Then $I_{\mathcal{P}_{G}}$ is generated by all the binomials $f_{\Gamma}$, where $\Gamma$ is an even closed walk of $G$. In particular, $I_{\mathcal{P}_{G}}=(0)$ if and only if each connected component of $G$ has at most one cycle and the cycle is odd.

The following theorem implies that the set of binomial generators of $I_{\mathcal{Q}_{G}}$ can also be characterized by the graph-theoretical terminology if $\alpha(G)=2$.

Theorem 3. Let $G$ be a simple graph with $\alpha(G)=2$. Then, $I_{\mathcal{Q}_{G}}=I_{\mathcal{P}_{\bar{G}}}+J$ where $J$ is an ideal generated by quadratic binomials $f_{\Gamma}$ where $\Gamma$ is an even closed walk of $\bar{G}^{\star}$ that satisfies one of the following:

(i) $\Gamma=(\{i, j\},\{j, k\},\{k, n+1\},\{n+1, i\})$ is a cycle where $\{i, j\},\{j, k\} \in E(\bar{G})$;

(ii) $\Gamma=(\{i, j\},\{j, n+1\},\{n+1, n+1\},\{n+1, i\})$ where $\{i, j\} \in E(\bar{G})$.

In particular, $d\left(I_{\mathcal{Q}_{G}}\right)=\max \left\{d\left(I_{\mathcal{P}_{\bar{G}}}\right), 2\right\}$.

Proof. Since $\alpha(G)=2$, we have $I_{\mathcal{Q}_{G}}=I_{\mathcal{P}_{\bar{G}^{\star}}}$ by Lemma 1 . Since $\bar{G}$ is a subgraph of $\bar{G}^{\star}$, it follows that $I_{\mathcal{P}_{\bar{G}^{\star}}} \supset I_{\mathcal{P}_{\bar{G}}}+J$. Thus, it is enough to show that $I_{\mathcal{P}_{\bar{G}^{\star}}} \subset I_{\mathcal{P}_{\bar{G}}}+J$.

Let $\Gamma$ be an even closed walk of $\bar{G}^{\star}$. It is enough to show that $f_{\Gamma} \in I_{\mathcal{P}_{\bar{G}^{\star}}}$ belongs to $I_{\mathcal{P}_{\bar{G}}}+J$. Suppose that $f_{\Gamma}$ does not belong to $I_{\mathcal{P}_{\bar{G}}}+J$. Then, the vertex $n+1$ belongs to $\Gamma$. We may assume that the degree of $f_{\Gamma}$ is minimum among binomials in $I_{\mathcal{P}_{\bar{G}^{\star}}}$ that do not belong to $I_{\mathcal{P}_{\bar{G}}}+J$. Then, $f_{\Gamma}$ is irreducible. Let

$$
\Gamma=\left(\{n+1, p\},\{p, q\},\{q, r\}, \Gamma^{\prime}\right),
$$

where $\Gamma^{\prime}=\left(e_{i_{1}}, \ldots, e_{i_{2 k+1}}\right)$ is an odd subwalk of $\Gamma$ from the vertex $r$ to the vertex $n+1$. Since $f_{\Gamma}$ is irreducible, it follows that $p, q, r$ are distinct vertices and that $q \neq n+1$. Then,

$$
f_{\Gamma}=\left(\prod_{\alpha=1}^{k} y_{i_{2 \alpha}}\right) f_{\Gamma_{1}}+y_{j} f_{\Gamma_{2}},
$$

where $e_{j}=\{p, q\}, \Gamma_{1}=(\{n+1, p\},\{p, q\},\{q, r\},\{r, n+1\})$, and $\Gamma_{2}=\left(\{n+1, r\}, \Gamma^{\prime}\right)$. Since $\operatorname{deg} f_{\Gamma_{2}}<$ $\operatorname{deg} f_{\Gamma}$, the binomial $f_{\Gamma_{2}}$ belongs to $I_{\mathcal{P}_{\bar{G}}}+J$ by the assumption on $\operatorname{deg} f_{\Gamma}$. Moreover, $\Gamma_{1}$ satisfies one of the conditions (i) or (ii), and hence $f_{\Gamma_{1}} \in J$. Thus, we have $f_{\Gamma} \in\left\langle f_{\Gamma_{1}}, f_{\Gamma_{2}}\right\rangle \subset I_{\mathcal{P}_{\bar{G}}}+J$, a contradiction. 
Remark 1. A graph-theoretical characterization of a simple graph G such that $d\left(I_{\mathcal{P}_{G}}\right) \leq 2$ is given in [5]. Then, by making use of Theorem 3, one can provide a similar characterization of a simple graph $G$ where $\alpha(G)=2$ and $d\left(I_{\mathcal{Q}_{G}}\right) \leq 2$.

It is known by ([11] Theorem 7.4) that if the complement of a graph $G$ is an even cycle of length $2 k$, then we have $d\left(I_{\mathcal{Q}_{G}}\right)=k$. By Theorem 3, we can generalize this result for a graph whose complement is an arbitrary bipartite graph.

Corollary 1. Let $G$ be a simple graph such that $\bar{G}$ is bipartite. Then we have

$$
d\left(I_{\mathcal{Q}_{G}}\right)= \begin{cases}0 & \text { if } \bar{G} \text { is empty (i.e., } G \text { is complete), } \\ k & \text { if } \bar{G} \text { has a cycle, } \\ 2 & \text { otherwise, }\end{cases}
$$

where $2 k$ is the maximum length of induced cycles of $\bar{G}$. Moreover, the following conditions are equivalent:

(i) $\quad d\left(I_{\mathcal{Q}_{G}}\right) \leq 2$, i.e., $I_{\mathcal{Q}_{G}}$ is generated by quadratic binomials;

(ii) $K\left[\mathcal{Q}_{G}\right]$ is Koszul;

(iii) $I_{\mathcal{Q}_{G}}$ has a quadratic Gröbner basis;

(iv) the length of any induced cycle of $\bar{G}$ is 4 .

Proof. Let $G$ be a simple graph such that $\bar{G}$ is bipartite. Then $\alpha(G)=2$. Since $\bar{G}$ is bipartite, it is known (see [23] Lemma 2.4) that $I_{\mathcal{P}_{\bar{G}}}$ is generated by $f_{\Gamma}$ where $\Gamma$ is an induced even cycle of $\bar{G}$. Note that $\operatorname{deg} f_{\Gamma}=k$ if the length of $\Gamma$ is $2 k$. Hence, by Theorem 3, we obtain the desired formula for $d\left(I_{\mathcal{Q}_{G}}\right)$.

It follows from the formula of $d\left(I_{\mathcal{Q}_{G}}\right)$ that (i) and (iv) are equivalent. Moreover, (iii) $\Rightarrow$ (ii) $\Rightarrow$ (i) holds in general. By Lemma 1 , there exists a bipartite graph $H$ such that $K\left[\mathcal{Q}_{G}\right] \simeq K\left[\mathcal{P}_{H}\right]$. By ([24] Theorem), $I_{\mathcal{P}_{H}}$ has a quadratic Gröbner basis if and only if $d\left(I_{\mathcal{P}_{H}}\right) \leq 2$. Thus, we have (i) $\Rightarrow$ (iii).

If $\bar{G}$ is not bipartite, then condition (i) and (iii) in Corollary 1 are not equivalent. In order to construct an infinite family of counterexamples, the following proposition is important. (Proof is essentially given in ([5] Proof of Proposition 1.6)).

Proposition 10. Let $\mathcal{P}$ be a $(0,1)$-polytope. If $I_{\mathcal{P}}$ has a quadratic Gröbner basis, then the initial ideal is generated by squarefree monomials, and hence $\mathcal{P}$ is normal.

Theorem 4. Let $G$ be a simple graph such that $\bar{G}=C_{1} \cup C_{2}$, where $C_{1}$ and $C_{2}$ are odd holes without common vertices. Then $\alpha(G)=2$, and

(a) $I_{\mathcal{Q}_{G}}$ is generated by quadratic binomials;

(b) $I_{\mathcal{Q}_{G}}$ has no quadratic Gröbner bases;

(c) $\mathcal{Q}_{G}$ is not normal.

Proof. Since $\bar{G}$ has no triangles, we have $\alpha(G)=2$. Since each connected component of $\bar{G}$ is an odd cycle, $I_{\mathcal{P}_{\bar{G}}}=\{0\}$ by Proposition 9. It follows from Theorem 3 that $I_{\mathcal{Q}_{G}}$ is generated by quadratic binomials. By Theorem $1, \mathcal{Q}_{G}$ is not normal since $C_{1}$ and $C_{2}$ have no bridges. Thus, by Proposition 10, $I_{\mathcal{Q}_{G}}$ has no quadratic Gröbner bases.

The graphs in Theorem 4 are not strongly Koszul by ([9] Theorem 1.3). However, we do not know whether they are Koszul or not in general.

Remark 2. It is known by ([5] Theorem 1.2) that if $G$ is a simple connected graph and $I_{\mathcal{P}_{G}}$ is generated by quadratic binomials, then $G$ satisfies the odd cycle condition, and hence $\mathcal{P}_{G}$ is normal. 
It seems to be a challenging problem to characterize the graphs $G$ such that $\alpha(G)>2$ and $d\left(I_{\mathcal{Q}_{G}}\right) \leq 2$. The following is a consequence of Proposition 6, Theorem 3 and ([5] Theorem 1.2).

Proposition 11. Let $G$ be a simple graph. If $I_{\mathcal{Q}_{G}}$ is generated by quadratic binomials, then $\bar{G}$ satisfies the following conditions:

(i) Any even cycle of $\bar{G}$ of length $\geq 6$ has a chord;

(ii) Any two odd holes of $\bar{G}$ having exactly one common vertex have a bridge;

(iii) Any two odd holes of $\bar{G}$ having no common vertex have at least two bridges.

Proof. Suppose that $\bar{G}$ does not satisfy one of the conditions above. If $\bar{G}$ does not satisfy condition (i), then let $H$ be an induced subgraph of $G$ whose vertex set is that of the even cycle. If $\bar{G}$ does not satisfy either condition (ii) or (iii), then let $H$ be an induced subgraph of $G$ whose vertex set is that of two odd holes. Then $\alpha(H)=2$, and hence $I_{\mathcal{Q}_{H}}$ is not generated by quadratic binomials by Theorem 3 and ([5] Theorem 1.2). Thus, it follows from Proposition 6 that $I_{\mathcal{Q}_{G}}$ is not generated by quadratic binomials.

Author Contributions: All authors made equal and significant contributions to writing this article, and approved the final manuscript.

Funding: The authors were partially supported by JSPS KAKENHI 17K14165 and 18H01134.

Acknowledgments: The authors are grateful to the anonymous referees for their careful reading and helpful comments.

Conflicts of Interest: The authors declare no conflict of interest.

\section{References}

1. Sturmfels, B. Gröbner Bases and Convex Polytopes; American Mathematical Society: Providence, RI, USA, 1996.

2. Herzog, J.; Hibi, T.; Ohsugi, H. Binomial Ideals; Graduate Texts in Math 279; Springer International Publishing: Cham, Switzerland, 2018.

3. Ohsugi, H.; Hibi, T. Convex polytopes all of whose reverse lexicographic initial ideals are squarefree. Proc. Am. Math. Soc. 2001, 129, 2541-2546. [CrossRef]

4. Sullivant, S. Compressed polytopes and statistical disclosure limitation. Tohoku Math. J. 2006, 58, 433-445. [CrossRef]

5. Ohsugi, H.; Hibi, T. Toric ideals generated by quadratic binomials. J. Algebra 1999, 218, 509-527. [CrossRef]

6. Sturmfels, B. Four counterexamples in combinatorial algebraic geometry. J. Algebra 2000, 230, $282-294$. [CrossRef]

7. Gouveia, J.; Parrilo, P.A.; Thomas, R.R. Theta bodies for polynomial ideals. SIAM J. Optim. 2010, 20, $2097-2118$. [CrossRef]

8. Ohsugi, H.; Hibi, T. Special simplices and Gorenstein toric rings. J. Combin. Theory Ser. A 2006, 113, 718-725. [CrossRef]

9. Matsuda, K. Strong Koszulness of toric rings associated with stable set polytopes of trivially perfect graphs. J. Algebra Appl. 2014, 13, 1350138. [CrossRef]

10. Hibi, T.; Li, N. Chain polytopes and algebras with straightening laws. Acta Math. Vietnam. 2015, 40, 447-452. [CrossRef]

11. Engström, A.; Norén, P. Ideals of graph homomorphisms. Ann. Comb. 2013, 17, 71-103. [CrossRef]

12. Sullivant, S. Toric fiber products. J. Algebra 2007, 316, 560-577. [CrossRef]

13. Gitler, I.; Reyes, E.; Villarreal, R.H. Ring graphs and toric ideals. Electron. Notes Discrete Math. 2007, 28, 393-400. [CrossRef]

14. Gitler, I.; Reyes, E.; Villarreal, R.H. Ring graphs and complete intersection toric ideals. Discrete Math. 2010, 310, 430-441. [CrossRef]

15. Diestel, R. Graph Theory, 4th ed.; Graduate Texts in Mathematics 173; Springer International Publishing: Berlin, Germany, 2010.

16. Ohsugi, H.; Hibi, T. Centrally symmetric configurations of integer matrices. Nagoya Math. J. 2014, 216, $153-170$. [CrossRef] 
17. Ohsugi, H.; Herzog, J.; Hibi, T. Combinatorial pure subrings. Osaka J. Math. 2000, 37, 745-757.

18. Ohsugi, H.; Hibi, T. Normal polytopes arising from finite graphsn. J. Algebra 1998, 207, 409-426. [CrossRef]

19. Simis, A.; Vasconcelos, W.V.; Villarreal, R.H. The integral closure of subrings associated to graphs. J. Algebra 1998, 199, 281-289. [CrossRef]

20. Ohsugi, H.; Hibi, T. A normal (0,1)-polytope none of whose regular triangulations is unimodular. Discrete Comput. Geom. 1999, 21, 201-204. [CrossRef]

21. Ohsugi, H. Toric ideals and an infinite family of normal $(0,1)$-polytopes without unimodular regular triangulations. Discrete Comput. Geom. 2002, 27, 551-565. [CrossRef]

22. Villarreal, R. Rees algebras of edge ideals. Commun. Algebra 1995, 23, 3513-3524. [CrossRef]

23. Simis, A. On the Jacobian module associated to a graph. Proc. Am. Math. Soc. 1998, 126, 989-997. [CrossRef]

24. Ohsugi, H.; Hibi, T. Koszul bipartite graphs. Adv. Appl. Math. 1999, 22, 25-28. [CrossRef]

(C) 2019 by the authors. Licensee MDPI, Basel, Switzerland. This article is an open access article distributed under the terms and conditions of the Creative Commons Attribution (CC BY) license (http://creativecommons.org/licenses/by/4.0/). 